\title{
Influência da idade e do peso no início do teste na análise genética de características de crescimento, reprodução e escores visuais de tourinhos Nelore em provas de ganho em peso a pasto
}

\author{
Tineo, J.S.A.; Raidan, F.S.S.; Santos, D.C.C. e Toral, F.L.B. ${ }^{\circledR}$
}

Universidade Federal de Minas Gerais. Belo Horizonte. MG. Brasil.

PALAVRAS CHAVE ADICIONAIS

Covariável.

Herdabilidade.

Melhoramento genético.

Seleç̃̃o.
INFORMACIÓN

Cronología del artículo.

Recibido/Received: 12.9.2013

Aceptado/Accepted: 13.5.2015

On-line: 16.03.2016

Correspondencia a los autores/Contact e-mail:

fllbtoral@ufmg.br

ADDITIONAL KEYWORDS

Heritability.

Breeding.

Selection.

\section{INTRODUÇÃO}

A utilização de touros jovens e melhoradores é uma alternativa para reduzir o intervalo de gerações e aumentar o ganho genético anual em sistemas de produção de bovinos de corte. Uma alternativa para aumentar a oferta desse tipo de touros é realizar provas

\section{RESUMO}

Objetivou-se verificar a contribuição da idade e peso no início do teste na análise genética do peso aos 550 dias, ganho médio diário, coeficiente de regressão linear do peso em função da idade, perímetro escrotal, volume testicular, e os escores de estrutura, precocidade e musculosidade de tourinhos Nelore em provas de ganho em peso (PGP) a pasto. Foram realizadas análises unicaracterísticas para estimação dos parâmetros, com quatro modelos estatísticos. No primeiro, foram inclúídos os efeitos fixo de PGP e aleatório do valor genético aditivo. No segundo e terceiro também foram incluídos os efeitos da idade e peso no início do teste como covariáveis, respectivamente. No quarto modelo, foram incluídos os efeitos das duas covariáveis. As características de crescimento e reprodutivas apresentam herdabilidades moderadas e a seleção pode contribuir para modificar suas médias. As características de morfologia apresentam herdabilidades baixas e, dessa forma, a resposta à seleção pode ser lenta ao longo das gerações. As inclusões das covariáveis resultam em alterações nas variâncias, herdabilidades e valores genéticos. Os modelos com inclusão do peso e/ou idade inicial como covariáveis são parcimoniosos para avaliação genética de características de crescimento, reprodução e escores visuais de tourinhos Nelore em provas de ganho em peso a pasto.

The influence of age and weight at the beginning of test in the genetic analysis of growth, reproductive and visual scores of Nellore young bulls on pasture performance tests

\section{SUMMARY}

The objective of this study was to verify the impact of inclusion of initial age and weight as covariable in the analysis of body weight at 550 days of age, average daily weight gain, weight linear regression coefficient in relation to age, scrotal circumference, testicular volume, and visual scores for Nellore young bulls' body structure, precocity, and muscularity under pasture performance tests (PT). Single-trait analyses for the estimation of the parameters were carried out through four statistical models. In the first model, the fixed effect of PT and random additive genetic value were included. In the second and third model, the effects of initial age and weight were also included as covariates, respectively. In the fourth model, the effects of the two covariates were included. Growth and reproductive traits have moderate heritability and selection can contribute to changing the means of these traits. Morphological traits have low heritability, which suggests that selection is not as efficient as to modify its means. Covariates inclusion result in alterations in the variances, heritability and genetic values. Models with initial age and, or, initial weight as covariates are parsimonious for the genetic evaluation of Nellore young bulls' growth, reproductive and visual scores under pasture weight gaining tests. de ganho em peso (PGP). Essas provas permitem mensurar o desempenho de forma mais eficiente, classificar os animais participantes, disponibilizar tourinhos jovens provados, avaliar o potencial genético de animais com genealogia desconhecida e agregam valor aos indivíduos de melhor desempenho. Seu baixo custo em relação a outros testes permite a avaliação de um 
maior número de animais, o que melhora a intensidade de seleção realizada (Razook et al., 1997). Dessa forma, a PGP está se tornando cada vez mais comum nos programas de melhoramento genético de bovinos de corte. Estudos para implantação de metodologias de avaliação genética mais adequada para os dados obtidos são importantes para aumentar a acurácia das avaliações e a resposta à seleção.

A metodologia de modelos mistos permite ajustar os dados fenotípicos para fatores não genéticos, como idade e peso no início do teste, e predizer os valores genéticos para características de interesse (Henderson et al., 1959). Animais com maior peso e idade no início do teste apresentam maior peso final (Gottschall et al., 2007 e Gottschall et al., 2009). Isso pode ser confirmado pela alta correlação genética $(0,67)$ observada entre o peso inicial e peso final de bovinos Nelore em PGP (Lima et al., 2013). Dessa forma, a inclusão dos efeitos da idade e peso inicial nos modelos de avaliação genética de bovinos de corte resultaria em maior acurácia na predição dos valores genéticos aditivos permitindo identificar e selecionar animais melhoradores com maior acurácia e aumentar o progresso genético e a produtividade nos sistemas de produção de bovinos de corte. De realçar ainda que a seleção pelos valores genéticos é um avanço em relação à seleção fenotípica, geralmente utilizada quando os animais são avaliados em PGP (Fragomeni et al., 2013). Dessa forma, objetivou-se verificar a contribuição da inclusão da idade e do peso no início do teste nos modelos estatísticos para análise genética de características de crescimento, reprodutivas e de morfologia de tourinhos Nelore em provas de ganho em peso a pasto.

\section{MATERIAL E MÉTODOS}

Os dados foram provenientes de 3193 animais Nelore, participantes de 42 provas de ganho em peso realizadas pelo grupo Provados a Pasto. O número de animais na matriz de parentesco foi 4 135. As provas foram realizadas entre 1997 e 2008 no estado de Goiás. Após a pesagem de entrada, houve um período de adaptação de 70 dias. Em seguida, foi realizada a pesagem inicial e o teste com duração de 221 dias, em média. Pesagens intermediárias, a cada 56 dias, também foram realizadas. A idade inicial variou de 283 a 429 dias.

Foram estudados: peso aos 550 dias (P550), ganho médio diário (GMD), coeficiente de regressão dos pesos em função da idade $(\hat{b})$, perímetro escrotal (PE), volume testicular (VT) e os escores visuais de estrutura corporal (E), precocidade (P) e musculosidade (M).

O P550 foi obtido por meio da equação

$$
P 550=P_{F}+\left(550-I_{F}\right) \times G M D ，
$$

em que: $P_{F}$ representa o peso real ao final da prova; $I_{F}$ a idade ao final da prova; e GMD, o ganho médio diário. O GMD foi obtido por meio da equação

$$
G M D=\frac{P_{F}-P_{I}}{I_{F}-I_{I}},
$$

em que: $P_{I}$ representa o peso ao início (após adaptação) e $I_{I}$ representa a idade ao início da prova.

Os valores de $\hat{b}$ foram obtidos pelo método de quadrados mínimos, regredindo-se os pesos ao longo da prova (peso inicial, pesos intermediários e peso final) em função da idade na pesagem, para cada animal com pelo menos quatro pesagens.

As medidas de PE foram realizadas no final da prova com fita métrica, posicionada transversalmente, na região de maior diâmetro da bolsa escrotal. Os comprimentos e larguras de cada testículo foram obtidos com paquímetro e, nesses casos, o VT foi estimado por meio da equação

$$
V T=0,5236 \times\left(C_{d} \times L_{d}^{2}+C_{e} \times L_{e}^{2}\right)
$$

em que: $C_{d}$ e $C_{e}$ representam os comprimentos dos testículos direito e esquerdo, respectivamente; e $L_{d}$ e $L_{e}$ representam as larguras dos testículos direito e esquerdo, respectivamente (Bailey et al., 1998).

Os escores visuais de E, P e M, na escala de 1 a 6, foram atribuídos a cada animal no final da prova, de acordo com técnica descrita em Koury Filho et al. (2009).

Os dados foram analisados por meio de quatro modelos estatísticos, sendo eles:

$$
\begin{aligned}
& y_{i l}=\mu+P G P_{i}+a_{l}+e_{i l} \\
& y_{i j l}=\mu+P G P_{i}+c_{(i)}\left(I_{I j}-\bar{I}_{I(i)}\right)+a_{l}+e_{i j l l} \\
& y_{i k l}=\mu+P G P_{i}+d_{(i)}\left(P_{I k}-\bar{P}_{I(i)}\right)+a_{l}+e_{i k l}, \mathrm{e} \\
& y_{i k l}=\mu+P G P_{i}+c_{(i)}\left(I_{i j}-\bar{I}_{I(i)}\right)+d_{(i)}\left(P_{I k}-\bar{P}_{I(i)}\right)+a_{l}+e_{i j k l}
\end{aligned}
$$

em que: $y$ (e subescritos) representa o fenótipo da característica; $\mu$, uma constante geral; $P G P_{i}$, o efeito fixo da prova de ganho em peso $i ; c_{(i)}$, o coeficiente de regressão da idade inicial sobre a característica $y$, aninhado em PGP; $I_{I j}$, a idade inicial $j ; \bar{I}_{I(i)}$, a média da idade inicial na prova $i ; d_{(i)}$, o coeficiente de regressão do peso inicial sobre a característica $y$, aninhado em PGP; $P_{I k}$, o peso inicial $k ; \bar{P}_{I(i)}$, a média do peso inicial na prova $i ; a_{l}$, o efeito aleatório genético aditivo do animal $l$; e $e$ (e subescritos), o erro aleatório.

Os componentes de variância foram estimados pelo método da máxima verossimilhança residual em análises unicaracterísticas, por meio do programa REMLF90 (Misztal, 2001). As contribuições das inclusões das covariáveis idade e peso inicial nos ajustes dos modelos foram verificadas pelo critério de informação de Akaike (AIC).

As correlações de Pearson e Spearman envolvendo os valores genéticos, a partir dos quatro modelos, dos animais que participaram da mesma prova foram calculadas para cada PGP. Posteriormente, obtiveram-se as médias das correlações envolvendo todas as provas. 
Tabela I. Estatísticas descritivas para as características de crescimento, reprodutivas e de morfologia de tourinhos Nelore em provas de ganho de peso a pasto (Descriptive statistics for Nellore young bulls' growth, reproductive and morphological traits under pasture performance tests).

\begin{tabular}{|c|c|c|c|c|c|c|c|c|}
\hline \multirow{2}{*}{ Estatística } & \multicolumn{8}{|c|}{ Características } \\
\hline & P550 & GMD & $b$ & PE & VT & E & $\mathrm{P}$ & $M$ \\
\hline N. Obs. & 3193 & 3193 & 2887 & 3010 & 600 & 802 & 915 & 915 \\
\hline N. PGP & 42 & 42 & 37 & 39 & 11 & 14 & 15 & 15 \\
\hline$I_{1}$ & 334,24 & 334,240 & 334,34 & 335,70 & 333,04 & 332,81 & 340,64 & 340,64 \\
\hline$P_{1}$ & 218,78 & 218,77 & 218,71 & 218,54 & 223,25 & 220,52 & 225,93 & 225,93 \\
\hline Média & 327,44 & 0,500 & 0,51 & 26,29 & 223,11 & 3,74 & 3,41 & 3,23 \\
\hline Desvio & 38,95 & 0,150 & 0,15 & 2,90 & 110,22 & 1,08 & 1,05 & 1,08 \\
\hline Mínimo & 186,79 & $-0,004$ & 0,03 & 18,00 & 44,00 & 1,00 & 1,00 & 1,00 \\
\hline Máximo & 466,82 & 0,960 & 0,91 & 39,00 & 692,72 & 6,00 & 6,00 & 6,00 \\
\hline CV $(\%)$ & 11,89 & 29,320 & 29,41 & 11,06 & 49,40 & 29,03 & 31,05 & 33,41 \\
\hline
\end{tabular}

N. Obs.= Número de observações; N. PGP= Número de provas de ganho em peso com registro da característica; $I_{1}=I_{d a d e}$ inicial (média, em dias); $P_{1}=$ Peso inicial (média, em kg); CV= Coeficiente de variação. P550= Peso aos 550 dias de idade (kg); GMD= Ganho médio diário $(\mathrm{kg} / \mathrm{dia}) ; b=$ Coeficiente de regressão linear do peso sobre a idade $(\mathrm{kg} / \mathrm{dia}) ; \mathrm{PE}=$ Perímetro escrotal $(\mathrm{cm}) ; \mathrm{VT}=$ Volume testicular $\left(\mathrm{cm}^{3}\right)$; $\mathrm{E}=$ Estrutura corporal (pontos); $\mathrm{P}=$ Precocidade (pontos); e $\mathrm{M}=$ Musculosidade (pontos).

Tabela II. Valores do critério de informação de Akaike nas análises de características de crescimento, reprodutivas e de morfologia de tourinhos Nelore em provas de ganho em peso a pasto, em modelos estatísticos sem ou com a inclusão das covariáveis peso $\left(\mathrm{P}_{\mathrm{I}}\right)$ e idade inicial $\left(\mathrm{I}_{\mathrm{I}}\right)$ (Akaike information criteria values for analysis of Nellore young bulls' reproductive and morphological traits under pasture performance tests, according to statistics models without or with the inclusion of weight $\left(P_{1}\right)$ and initial age $\left(l_{1}\right)$ covariates).

\begin{tabular}{lccccccccc}
\hline \multirow{2}{*}{ Covariáveis incluídas } & \multicolumn{7}{c}{ Características } \\
\cline { 2 - 9 } & $\mathrm{P} 550$ & GMD & $\hat{b}$ & PE & VT & E & P & M \\
\hline- & 31418 & -5679 & -4412 & 15585 & 8140 & 7990 & 7978 & 8037 \\
$\mathrm{I}_{1}$ & 31444 & -6418 & -4507 & 13703 & 6723 & 3612 & 6766 & 6830 \\
$\mathrm{P}_{1}$ & 28876 & -5122 & -3925 & 15461 & 13410 & 7748 & 6650 & 7926 \\
$\mathrm{I}_{1}+\mathrm{P}_{\mathrm{I}}$ & 28391 & -4639 & -5834 & 15186 & 6693 & 4552 & 6770 & 6771 \\
\hline
\end{tabular}

$\mathrm{P}_{\mathrm{I}}=$ Peso inicial (média, em kg); $\mathrm{l}_{1}=$ Idade inicial (média, em dias); $\mathrm{P} 550=$ Peso aos 550 dias de idade; GMD= Ganho médio diário; $\hat{b}=$ Coeficiente de regressão linear do peso sobre a idade; $\mathrm{PE}=$ Perímetro escrotal; $\mathrm{VT}=$ Volume testicular; $\mathrm{E}=\mathrm{Estrutura}$ corporal; $\mathrm{P}=\mathrm{Precocidade}$; e $M=$ Musculosidade.

\section{RESULTADOS E DISCUSSÃO}

Em geral, as médias observadas para as características estudadas (tabela I) estão dentro do intervalo observado na raça Nelore (Boligon et al., 2010; Raidan et al., 2015), demostrando que o banco de dados utilizado pode ser considerado representativo da raça.

O critério AIC foi mais próximo de zero para modelos estatísticos com inclusão de covariáveis idade e/ ou, peso inicial (tabela II). Portanto, estas covariáveis explicam parte significativa das variações observadas nas características analisadas. A inclusão da idade e peso inicial nos modelos estatísticos do P550, GMD, VT e $\mathrm{M}$ resultou em melhoria significativa no ajuste dos modelos. No caso de $\hat{b}$ e P, a inclusão do peso inicial, apenas, foi suficiente para melhorar o ajuste. Para as variáveis PE e E, a inclusão da idade inicial, juntamente com os outros efeitos comuns, resultou em melhores ajustamentos.

Outros estudos reportaram a inclusão da idade no dia da mensuração como covariável na análise do P550 (Kippert et al., 2008), ganho médio diário pós-desmama (Kippert et al., 2008; Queiroz et al., 2013), PE (Lopes et al., 2009), VT (Boligon et al., 2010) e escores de conformação (C), E, P e M (Koury Filho et al., 2009; Queiroz et al., 2013) em bovinos de corte, demonstrando a importância desse fator.

Para características que não foram ajustadas para uma idade de referência (PE, VT, E, P e M), a inclusão do efeito da idade e/ou do peso, é fundamental para evitar que diferenças ambientais prejudiquem a avaliação genética. Diferenças no peso inicial podem ocorrer, por exemplo, por diferenças nos manejos que antecedem os testes ou por diferenças genéticas entre rebanhos. Tais diferenças, dependendo da magnitude, podem permanecer após o período de adaptação e prejudicar a classificação dos animais se elas não forem modeladas.

Mesmo para o estudo de características padronizadas para uma idade de referência (P550 e GMD), ainda é necessário incluir o efeito da covariável idade no modelo. Isso demonstra que o ajuste linear, apenas, não é suficiente para retirar todo o efeito da idade sobre o crescimento e sobre características reprodutivas e de morfologia. Essa necessidade pode ser explicada pelo comportamento não linear do peso (Carneiro et al., 2014) e do PE (Loaiza-Echeverri et al., 2013) em função da idade. 
Tabela III. Estimativas de variâncias genéticas aditivas $\left(\hat{\sigma}_{a}^{2}\right)$ e residuais $\left(\hat{\sigma}_{e}^{2}\right)$ e herdabilidade $\left(\hat{h}^{2}\right)$ para características de crescimento, reprodutivas e de morfologia de tourinhos Nelore em provas de ganho em peso a pasto, em modelos estatísticos sem ou com a inclusão das covariáveis peso $\left(\mathrm{P}_{\mathrm{I}}\right)$ e idade inicial $\left(\mathrm{I}_{\mathrm{I}}\right)$ (Estimates of aditive genetic $\left(\hat{\sigma}_{a}^{2}\right)$ and residual $\left(\hat{\sigma}_{e}^{2}\right)$ variances and heritability $\left(\hat{h}^{2}\right)$ for Nellore young bulls' growth, reproductive and morphological traits under pasture performance tests, according to statistics models without or with the inclusion of weight $\left(\mathrm{P}_{\mathrm{I}}\right)$ and initial age $\left(\mathrm{I}_{1}\right)$ covariates).

\begin{tabular}{|c|c|c|c|c|c|c|c|c|}
\hline \multirow{2}{*}{$\begin{array}{l}\text { Covariáveis } \\
\text { incluídas }\end{array}$} & \multicolumn{8}{|c|}{ Características } \\
\hline & P550 & GMD & $\hat{b}$ & PE & VT & $E$ & $\mathrm{P}$ & M \\
\hline & \multicolumn{8}{|c|}{$\hat{\sigma}_{a}^{2}$} \\
\hline- & 626,50 & 0,002 & 0,0200 & 3,24 & 1917 & 0,14 & 0,21 & 0,09 \\
\hline$I_{1}$ & 595,10 & 0,002 & 0,0021 & 2,10 & 2107 & 0,15 & 0,19 & 0,07 \\
\hline$P_{1}$ & 147,90 & 0,001 & 0,0167 & 3,44 & 1703 & 0,06 & 0,14 & 0,07 \\
\hline \multirow[t]{2}{*}{$\mathrm{I}_{1}+\mathrm{P}_{1}$} & 77,85 & 0,002 & 0,0041 & 2,94 & 1700 & 0,01 & 0,13 & 0,05 \\
\hline & \multicolumn{8}{|c|}{$\hat{\sigma}_{e}^{2}$} \\
\hline - & 258,20 & 0,005 & 0,0439 & 4,00 & 4559 & 0,90 & 0,70 & 0,87 \\
\hline$I_{1}$ & 262,60 & 0,004 & 0,0041 & 3,16 & 2978 & 0,75 & 0,64 & 0,81 \\
\hline$P_{1}$ & 205,70 & 0,005 & 0,0455 & 2,97 & 3775 & 0,63 & 0,58 & 0,65 \\
\hline \multirow[t]{2}{*}{$\mathrm{I}_{1}+\mathrm{P}_{1}$} & 204,10 & 0,004 & 0,0041 & 2,21 & 2997 & 0,62 & 0,58 & 0,66 \\
\hline & \multicolumn{8}{|c|}{$\hat{h}^{2}$} \\
\hline- & 0,71 & 0,28 & 0,31 & 0,45 & 0,30 & 0,13 & 0,23 & 0,10 \\
\hline$I_{1}$ & 0,69 & 0,29 & 0,34 & 0,40 & 0,41 & 0,17 & 0,23 & 0,09 \\
\hline$P_{1}$ & 0,42 & 0,24 & 0,27 & 0,54 & 0,31 & 0,08 & 0,20 & 0,09 \\
\hline $\mathrm{I}_{1}+\mathrm{P}_{1}$ & 0,28 & 0,27 & 0,34 & 0,57 & 0,37 & 0,01 & 0,18 & 0,07 \\
\hline
\end{tabular}

$\mathrm{P}_{1}=$ Peso inicial (média, em kg); $\mathrm{I}_{1}=$ Idade inicial (média, em dias); $\mathrm{P} 550=\mathrm{Peso}$ aos 550 dias de idade; $\mathrm{GMD}=$ Ganho médio diário; $\hat{b}=$ Coeficiente de regressão linear do peso sobre a idade; $P E=$ Perímetro escrotal; $V T=$ Volume testicular; $E=E$ Estrutura corporal; $P=P r e c o c i d a d e ;$ e $M=$ Musculosidade.

A inclusãa do efeito da idade inicial na análise do coeficiente $\hat{b}$ prejudicou o ajuste do modelo, enquanto a inclusão do peso inicial, isoladamente, teve efeito contrário. As idades de pesagens (inclusive a idade inicial) foram consideradas como variáveis independentes na obtenção de $\hat{b}$. Portanto, a inclusão dessa covariável, novamente, pode ter causado multicolinearidade.

As inclusões das covariáveis peso e idade inicial, separadamente, resultaram em modelos melhor ajustados aos dados de precocidade. Entretanto, o ajuste proporcionado pela inclusão dessas covariáveis ao mesmo tempo não foi melhor que o ajuste proporcionado pela inclusão apenas do peso.

Não foram encontrados relatos na literatura sobre a inclusão do peso inicial como covariável para análise das características de desempenho em PGP. Há trabalhos demonstrando a necessidade de inclusão do efeito do peso no momento da mensuração sobre o PE em bovinos de corte (Peña et al., 2000). No presente trabalho, a avaliação da inclusão dessa covariável foi realizada para viabilizar o atendimento de uma condição importante para realização das PGP, ou seja, animais com pesos e idades semelhantes (Razook et al., 1997).

A homogeneidade relacionada com a idade é garantida, parcialmente, pelo intervalo máximo de idade na PGP de 90 dias (Josahkian et al., 2009). Entretanto, não há restrição relacionada ao peso de entrada. Considerando a dificuldade para obtenção de animais com pesos de entrada semelhantes, a inclusão da covariável peso inicial nos modelos permitiria avaliar o desem- penho dos animais em PGP a partir de uma referência de peso constante.

Em geral, a inclusão das covariáveis peso e/ou idade inicial nos modelos estatísticos provocaram reduções nas variâncias e alterações na herdabilidade (tabela III). As estimativas de variâncias genéticas foram menores nos modelos de melhor ajuste, em relação ao modelo mais simples, exceto para GMD e E. Neves et al. (2014) verificaram que a inclusão do efeito aleatório de rebanho de origem reduziu as estimativas de variância genética aditiva para peso ao final da PGP de tourinhos Nelore. O efeito de rebanho de origem está relacionado com o peso de entrada nos animais o que, de certa forma, é semelhante à covariável analisada no presente trabalho (peso inicial). No caso de P550, a inclusão do efeito das covariáveis peso e idade inicial resultaram em estimativas de herdabilidade praticamente iguais aquelas obtidas com o mesmo modelo para GMD e $b$. Quando o P550 é ajustado para um mesmo peso inicial, diferenças nessa variável são reflexos de diferenças no ganho médio diário.

As estimativas de herdabilidade para GMD, PE e VT em tourinhos Nelore avaliados em PGP a pasto foram semelhantes aos valores de 0,26 (Raidan et al., 2015); 0,42 e 0,39 (Boligon et al., 2010), respectivamente, observados em condições semelhantes. De modo geral, essas características possuem herdabilidade moderada e é possível obter mudanças genéticas na média da população por meio da seleção.

As estimativas de herdabilidade para E, $\mathrm{P}$ e $\mathrm{M}$ foram baixas e inferiores aos valores de 0,24, 0,63 e 0,48, respectivamente, obtidos por Koury Filho et al. (2009). 
Tabela IV. Médias de correlações de Pearson e Spearman entre os valores genéticos para características de crescimento, reprodutivas e de morfologia de tourinhos Nelore em provas de ganho em peso a pasto, em modelos estatísticos sem ou com a inclusão das covariáveis peso $\left(\mathrm{P}_{\mathrm{I}}\right)$ e idade inicial $\left(\mathrm{I}_{\mathrm{I}}\right)$ (Means for Pearson and Spearman correlations among breeding values for Nellore young bulls' growth, reproductive and morphological traits under pasture performance tests, according to statistics models without or with inclusion of weight $\left(P_{1}\right)$ and initial age $\left(I_{1}\right)$ covariates).

\begin{tabular}{|c|c|c|c|c|c|c|c|c|c|}
\hline \multirow{2}{*}{$\begin{array}{l}\text { Covariáveis } \\
\text { incluídas }\end{array}$} & \multirow{2}{*}{$\begin{array}{l}\text { Covariáveis } \\
\text { incluídas }\end{array}$} & \multicolumn{8}{|c|}{ Características } \\
\hline & & P550 & GMD & $\hat{b}$ & $\mathrm{PE}$ & VT & $E$ & $\mathrm{P}$ & M \\
\hline & & \multicolumn{8}{|c|}{ Correlação de Pearson } \\
\hline- & $\mathrm{I}_{1}$ & 0,98 & 0,98 & 0,98 & 0,84 & 0,82 & 0,92 & 0,96 & 0,95 \\
\hline- & $P_{1}$ & 0,58 & 0,98 & 0,99 & 0,91 & 0,89 & 0,69 & 0,88 & 0,84 \\
\hline- & $\mathrm{I}_{1}+\mathrm{P}_{1}$ & 0,58 & 0,96 & 0,97 & 0,83 & 0,85 & 0,71 & 0,88 & 0,83 \\
\hline$I_{1}$ & $P_{1}$ & 0,60 & 0,97 & 0,98 & 0,79 & 0,95 & 0,68 & 0,88 & 0,82 \\
\hline $\mathrm{I}_{1}$ & $\mathrm{I}_{1}+\mathrm{P}_{1}$ & 0,60 & 0,98 & 0,98 & 0,98 & 0,91 & 0,74 & 0,91 & 0,85 \\
\hline \multirow[t]{2}{*}{$\mathrm{P}_{1}$} & $\mathrm{I}_{1}+\mathrm{P}_{1}$ & 1,00 & 0,97 & 0,98 & 0,79 & 0,96 & 0,98 & 0,99 & 0,99 \\
\hline & & \multicolumn{8}{|c|}{ Correlação de Spearman } \\
\hline- & $I_{1}$ & 0,97 & 0,97 & 0,98 & 0,81 & 0,77 & 0,90 & 0,94 & 0,94 \\
\hline- & $P_{1}$ & 0,53 & 0,98 & 0,98 & 0,90 & 0,85 & 0,66 & 0,85 & 0,82 \\
\hline- & $\mathrm{I}_{1}+\mathrm{P}_{1}$ & 0,53 & 0,94 & 0,95 & 0,81 & 0,80 & 0,68 & 0,85 & 0,83 \\
\hline $\mathrm{I}_{1}$ & $P_{1}$ & 0,56 & 0,95 & 0,96 & 0,76 & 0,94 & 0,64 & 0,84 & 0,81 \\
\hline$I_{1}$ & $\mathrm{I}_{1}+\mathrm{P}_{1}$ & 0,56 & 0,97 & 0,97 & 0,97 & 0,89 & 0,70 & 0,88 & 0,84 \\
\hline$P_{1}$ & $\mathrm{I}_{1}+\mathrm{P}_{1}$ & 1,00 & 0,96 & 0,96 & 0,77 & 0,95 & 0,98 & 0,98 & 0,98 \\
\hline
\end{tabular}

$\mathrm{P}_{1}=$ Peso inicial (média, em kg); $\mathrm{l}_{1}=$ Idade inicial (média, em dias); $\mathrm{P} 550=$ Peso aos 550 dias de idade; GMD= Ganho médio diário; $\hat{b}=$ Coeficiente de regressão linear do peso sobre a idade; $P E=$ Perímetro escrotal; VT= Volume testicular; $E=E$ Estrutura corporal; $P=P r e c o c i d a d e ;$ e $M=$ Musculosidade.

Assim, espera-se pouca resposta à seleção para estas características no grupo de animais avaliados. Mesmo considerando diferenças genéticas entre as populações avaliadas, as diferenças observadas nas estimativas de herdabilidade foram altas e podem refletir, também, diferenças na qualidade da coleta de dados. Portanto, é recomendado que técnicos responsáveis por essas mensurações sejam treinados e realizem os procedimentos de forma padronizada.

A inclusão das covariáveis idade e/ou peso inicial nos modelos estatísticos para análise de características de crescimento, reprodutivas e de morfologia de tourinhos Nelore implicou em mudanças nas estimativas dos componentes de variância (tabela III) e nos valores genéticos preditos, como pode ser visualizado pelas correlações (tabela IV).

A inclusão das covariáveis peso e/ou idade inicial nos modelos resultaram em mudanças nos valores genéticos e na classificação dos tourinhos dentro de cada PGP. Esse resultado ficou mais evidente para P550, mas modificações também ocorreram para PE, VT, E, P e M. No caso de P550, a inclusão do peso inicial no modelo reduziu a variância genética aditiva em 80 \%. O peso no início do teste representa 70\% do P550. Então, se não houver ajuste das características de interesse para um peso inicial constante, grandes diferenças no início do teste podem determinar o resultado final da prova e, desse modo, reduzir a eficiência da PGP como ferramenta para identificação dos méritos genéticos dos animais.

\section{CONCLUSÕES}

A inclusão das covariáveis peso e/ou idade inicial proporciona modelos estatísticos parcimoniosos para análise do desempenho de características de crescimento, reprodução e escores visuais de tourinhos Nelore em provas de ganho em peso a pasto.

\section{BIBLIOGRAFIA}

Bailey, T.L.; Hudson, R.S.; Powe, T.A.; Riddell, M.G.; Wolfe, D.F. and Carson, R.L. 1998. Caliper and ultrasonographic measurements of bovine testicles and a mathematical formula for determining testicular volume and weight in vivo. Theriogenology, 49:581-594. http://www. ncbi.nlm.nih.gov/pubmed/10732037 (10/11/2014).

Boligon, A.A.; Silva, J.A.V.; Sesana, R.C.; Sesana, J.C.; Junqueira, J.B. and Albuquerque, L.G. 2010. Estimation of genetic parameters for body weights, scrotal circumference, and testicular volume measured at different ages in Nellore cattle. J Anim Sci, 88: 1215-1219. https:// dl. sciencesocieties.org/publications/jas/abstracts/88/4/1215 (01/12/2014).

Carneiro, A.P.S.; Muniz, J.A.; Carneiro, P.L.S.; Malhado, C.H.M.; Martins Filho, R. e Silva, F.F. 2014. Identidade de modelos não lineares para comparar curvas de crescimento de bovinos da raça Tabapuã. PesqAgropec Bras, 49: 57-62. http://www.scielo.br/scielo. php?pid=S0100-204X2014000100009\&script=sci_arttext\&tlng=es (05/12/2014).

Fragomeni, B.O.; Scalez, D.C.B.; Toral, F.L.B.; Bergmann, J.A.; Pereira, I.G. and Costa, P.S.T. 2013. Genetic parameters and alternatives for evaluation and ranking of Nellore young bulls in pasture performance tests. Rev Bras Zootecn, 42: 559-564. http://www.scielo.br/scielo. php?pid=S1516-35982013000800004\&script=sci_arttext\&tlng=pt (8/03/2014).

Gottschall, C.S.; Canellas, L.C.; Ferreira, E.T. e Bittencourt, H.R. 2007. Desempenho de novilhos Angus, Devon e cruzas Angus $x$ Devon $x$ Nelore em confinamento. Semin-Cienc Agrar, 28: 135-142. http:// 
www.uel.br/revistas/vel/index.php/semagrarias/article/view/2569 (23/02/2016).

Gottschall, C.S.; Canellas, L.C.; Marques, P.R. e Bittencourt, H.R. 2009. Relações entre idade, peso, ganho médio diário e tempo médio de permanência de novilhos de corte confinados para abate aos 15 ou 27 meses de idade. Semin-Cienc Agrar, 30: 717-726. http://www. vel.br/revistas/vel/index.php/semagrarias/article/view/3588 (23/02/2016).

Henderson, C.R.; Kempthorne, O.; Searle, S.R. and Von Krosigk, C.M. 1959. The estimation of environmental and genetic trends from records subject to culling. Biometrics, 15: 192-218. http://www.lce. esalq.usp.br/arquivos/aulas/2012/LCE5872/Henderson 1959.pdf (11/02/2015)

Josahkian, L.A.; Lucas, C.H. e Machado, C.H.C. 2009. Manual do Serviço de Registro Genealógico das Raças Zebuínas e PMGZ. Associação Brasileira dos Criadores de Zebu. Uberaba. 190 pp. http://www. abcz.org.br/Content/arquivos/AreaTecnica/SRGRZ/manual-do-reggenealogico-e-pmgz.pdf (23/02/2016).

Kippert, J.C.; Rorato, P.R.N.; Lopes, J.S.; Weber, T. e Boligon, A.A. 2008. Efeitos genéticos aditivos diretos e maternos e heterozigoticos sobre os desempenhos pré e pós-desmame em uma população multirracial Aberdeen Angus x Nelore. Rev Bras Zootecn, 37: 1383-1391.

Koury Filho, W.; Albuquerque, L.G.; Alencar, M.M.; Forni, S.; Silva, J.A.II.V. e Lôbo, R.B. 2009. Estimativas de herdabilidade e correlações para escores visuais, peso e altura ao sobreano em rebanhos da raça Nellore. Rev Bras Zootecn, 38: 2362-2367. http://www.scielo.br/ scielo.php?pid=S1516-35982009001200010\&script=sci_arttext (01/03/2014).

Lima, P.R.M.; Paiva, S.R.; Cobuci, J.A.; Braccini Neto, J.; Machado, C.H.C. and McManus, C. 2013. Genetic parameters for type classification of Nellore cattle on central performance tests at pasture. Brazil Trop Anim Health Prod, 45:1627-1634. http://www.ncbi.nlm.nih.gov/ pubmed/23568619 (23/02/2016).

Loaiza-Echeverri, A.M.; Bergmann, J.A.G.; Toral, F.L.B.; Osorio, J.P.; Carmo, A.S.; Mendonça, L.F.; Moustacas, V.S. and Henry, M. 2013. Use of nonlinear models for describing scrotal circumference growth in Guzerat bulls raised under grazing conditions. Theriogenology, 79: 751-759. http://www.sciencedirect.com/science/article/pii/ S0093691X12006395 (08/04/2013).

Lopes, J.S.; Rorato, P.R.N.; Weber, T.; Comin, J.G. e Araújo, R.O. 2009. Fatores de correção para perímetro escrotal ao sobreano para tourinhos mestiços Aberdeen Angus x Nelore. Arq Bras Med Vet Zootec, 61: 413-419. http://www.scielo.br/scielo.php?.pid=S0102$09352009000200019 \&$ script=sci_arttext (24/01/ 2013).

Misztal, I. 2001. REMLF90 Manual. http://nce.ads.uga.edu/ ignacy/ numpub/blupf90/docs/remlf90.pdf (06/05/2013).

Neves, H.H.R.; Reis, F.P.; Paterno, F.M.; Guarini, A.R.; Carvalheiro, R.; Silva, L.R.; Oliveira, J.A. and Queiroz, S.A. 2014. Herd-of-origin effect on the post-weaning performance of centrally tested Nellore beef cattle. Trop Anim Health Prod, 46: 1235-1241. http://link.springer.com/ article/10.1007/s1 1250-014-0633-2/fulltext.html (02/01/2015).

Peña, C.D.O.; Queiroz, S.A. e Fries, L.A. 2001. Comparação entre critérios de seleção de precocidade sexual e a associação destes com características decrescimento em bovinos Nelore. Rev Bras Zootecn, 30: 93-100. http://www.scielo.br/pdf/rbz/v30n1/5440. pdf (06/01/2013).

Queiroz, S.A.; Oliveira, J.A.; Costa, G.Z. e Fries, L.A. 2013. Efeitos ambientais e genéticos sobre escores visuais e ganho em peso ao sobreano de bovinos Brangus. Arch Zootec, 62: 111-121. https:// www.uco.es/organiza/servicios/publica/az/php/az.php?idioma_gl obal=0\&revista=174\&codigo=2174 (06/01/2013).

Raidan, F.S.S.; Passafaro, T.L.; Fragomeni, B.O.; Josahkian, L.A.; Pereira, I.G. and Toral, F.L.B. 2015. Genotype x environment interaction in individual performance and progeny tests in beef cattle. J Anim Sci, 93: 920-933. https://dl.sciencesocieties.org/publications/jas/ abstracts/93/3/920 (06/04/2015).

Razook, A.G.; Figueiredo, L.A.; Cyrillo, J.S.G.; Pacola, L.J.; Bonilha Neto, L.M.; Trovo, J.B.F.; Ruggieri, A.C. e Mercadante, M.E.Z. 1997. Prova de ganho de peso: normas adotadas pela Estação Experimental de Zootecnia de Sertãozinho. Instituto de Zootecnia. Nova Odessa. Boletín Técnico n 40 . http://www.iz.sp.gov.br/pdfs/1 195668031. $\operatorname{pdf}(23 / 02 / 2016)$. 